\title{
Refinements of the Heinz inequalities
}

\author{
Yuming Feng
}

Correspondence:

yumingfeng25928@163.com School of Mathematics and

Statistics, Chongqing Three Gorges University, Wanzhou, Chongqing

404100, P.R. China

\begin{abstract}
This article aims to discuss Heinz inequalities involving unitarily invariant norms. We obtain refinements of the Heinz inequalities. In particular, our results refine some results given in Kittaneh.
\end{abstract}

Keywords: refinements, Heinz inequality, convex function, Hermite-Hadamard inequality, unitarily invariant norm

\section{Introduction}

If $A, B, X$ are operators on a complex separable Hilbert space such that $A$ and $B$ are positive, then for every unitarily invariant norm $\||\cdot \||$, the function $f(v)=\||| A^{v} X B^{1-v}+A^{1-v} X B^{v} \| \mid$ is convex on the interval $[0,1]$, attains its minimum at $v=\frac{1}{2}$, and attains its maximum at $v=0$ and $v=1$. Moreover, $f(v)=f(1-v)$ for $0 \leq v \leq 1$. Thus, for every unitarily invariant norm, we have the Heinz inequalities (see [1])

$$
2\left|\left\|A^{\frac{1}{2}} X B^{\frac{1}{2}}\right\|\right| \leq\left|\left\|A^{v} X B^{1-v}+A^{1-v} X B^{v}\right\|\right| \leq|\|A X+X B\|| .
$$

In this article, we use the convexity of the function

$$
f(v)=\left|\left\|A^{v} X B^{1-v}+A^{1-v} X B^{v}\right\|\right|
$$

on $[0,1]$ to obtain new refinements of the inequalities (1.1). Our analysis enables us to discuss the equality conditions in (1.1) for certain unitarily invariant norms. When we consider $\||| T \mid\|$, we are implicitly assuming that the operator $T$ belongs to the norm ideal associated with \|\|$\cdot\|\|$. Our results are better than those in [2].

\section{Main results}

The following Hermite-Hadamard integral inequality for convex functions is well known (see p. 122 in [3], also see Lemma 1 in [2]).

Lemma 1 (Hermite-Hadamard Integral Inequality). Let $f$ be a real-valued function which is convex on the interval $[a, b]$. Then

$$
f\left(\frac{a+b}{2}\right) \leq \frac{1}{b-a} \int_{a}^{b} f(t) d t \leq \frac{f(a)+f(b)}{2} .
$$

In [2], Kittaneh obtained several refinements of the Heinz inequalities by using the previous lemma. In the following, we will use the following lemma to obtain several better refinements of the Heinz inequalities.

(c) 2012 Feng; licensee Springer. This is an Open Access article distributed under the terms of the Creative Commons Attribution License (http://creativecommons.org/licenses/by/2.0), which permits unrestricted use, distribution, and reproduction in any medium, provided the original work is properly cited. 
The following lemma can be proved by using the previous lemma.

Lemma 2. Let $f$ be a real-valued function which is convex on the interval $[a, b]$.

Then

$$
f\left(\frac{a+b}{2}\right) \leq \frac{1}{b-a} \int_{a}^{b} f(t) d t \leq \frac{1}{4}\left(f(a)+2 f\left(\frac{a+b}{2}\right)+f(b)\right) \leq \frac{f(a)+f(b)}{2}
$$

Proof. Using the previous lemma, we can easily verify the inequality

$$
\frac{1}{4}\left(f(a)+2 f\left(\frac{a+b}{2}\right)+f(b)\right) \leq \frac{f(a)+f(b)}{2} .
$$

Next, we will prove the following inequality.

$$
\frac{1}{b-a} \int_{a}^{b} f(t) d t \leq \frac{1}{4}\left(f(a)+2 f\left(\frac{a+b}{2}\right)+f(b)\right) .
$$

From the previous lemma, we have

$$
\begin{aligned}
\frac{1}{b-a} \int_{a}^{b} f(t) d t & =\frac{1}{b-a}\left(\int_{a}^{\frac{a+b}{2}} f(t) d t+\int_{\frac{a+b}{2}}^{b} f(t) d t\right) \\
& \leq \frac{1}{b-a}\left(\frac{f(a)+f\left(\frac{a+b}{2}\right)}{2} \cdot \frac{b-a}{2}+\frac{f\left(\frac{a+b}{2}\right)+f(a)}{2} \cdot \frac{b-a}{2}\right) \\
& =\frac{1}{4}\left(f(a)+2 f\left(\frac{a+b}{2}\right)+f(b)\right) .
\end{aligned}
$$

Applying the previous lemma to the function $f(v)=\left\||| A^{v} X B^{1-v}+A^{1-v} X B^{v}\right\| \|$ on the interval $[\mu, 1-\mu]$ when $0 \leq \mu \leq \frac{1}{2}$, and on the interval $[1-\mu, \mu]$ when $\frac{1}{2} \leq \mu \leq 1$, we obtain refinement of the first inequality in (1.1).

Theorem 1. Let $A, B, X$ be operators such that $A, B$ are positive. Then for $0 \leq \mu \leq 1$ and for every unitarily invariant norm, we have

$$
\begin{aligned}
2\left|\left\|A^{\frac{1}{2}} X B^{\frac{1}{2}}\right\|\right| & \leq \frac{1}{|1-2 \mu|}\left|\int_{\mu}^{1-\mu}\right|\left\|A^{v} X B^{1-v}+A^{1-v} X B^{v}\right\||d v| \\
& \leq \frac{1}{2}\left(\left|\left\|A^{\mu} X B^{1-\mu}+A^{1-\mu} X B^{\mu}\right\|\right|+2\left|\left\|A^{\frac{1}{2}} X B^{\frac{1}{2}}\right\|\right|\right) \\
& \leq\left|\left\|A^{\mu} X B^{1-\mu}+A^{1-\mu} X B^{\mu}\right\|\right| .
\end{aligned}
$$

Proof. First assume that $0 \leq \mu \leq \frac{1}{2}$. Then it follows by the previous lemma that

$$
\begin{aligned}
f\left(\frac{1-\mu+\mu}{2}\right) & \leq \frac{1}{1-2 \mu} \int_{\mu}^{1-\mu} f(t) d t \\
& \leq \frac{1}{4}\left(f(\mu)+2 f\left(\frac{1-\mu+\mu}{2}\right)+f(1-\mu)\right) \\
& \leq \frac{f(\mu)+f(1-\mu)}{2}
\end{aligned}
$$


and so

$$
\begin{aligned}
f\left(\frac{1}{2}\right) & \leq \frac{1}{1-2 \mu} \int_{\mu}^{1-\mu} f(t) d t \\
& \leq \frac{1}{2}\left(f(\mu)+f\left(\frac{1}{2}\right)\right) \\
& \leq f(\mu) .
\end{aligned}
$$

Thus,

$$
\begin{aligned}
2\left|\left\|A^{\frac{1}{2}} X B^{\frac{1}{2}}\right\|\right| & \leq \frac{1}{1-2 \mu} \int_{\mu}^{1-\mu}\left|\left\|A^{v} X B^{1-v}+A^{1-v} X B^{v}\right\|\right| d v \\
& \leq \frac{1}{2}\left(\left|\left\|A^{\mu} X B^{1-\mu}+A^{1-\mu} X B^{\mu}\right\|\right|+2\left|\left\|A^{\frac{1}{2}} X B^{\frac{1}{2}}\right\|\right|\right) \\
& \leq\left|\left\|A^{\mu} X B^{1-\mu}+A^{1-\mu} X B^{\mu}\right\|\right| .
\end{aligned}
$$

Now, assume that $\frac{1}{2} \leq \mu \leq 1$. Then by applying (2.2) to $1-\mu$, it follows that

$$
\begin{aligned}
2\left|\left\|A^{\frac{1}{2}} X B^{\frac{1}{2}}\right\|\right| & \leq \frac{1}{2 \mu-1} \int_{1-\mu}^{\mu}\left|\left\|A^{v} X B^{1-v}+A^{1-v} X B^{v}\right\|\right| d v \\
& \leq \frac{1}{2}\left(\left|\left\|A^{\mu} X B^{1-\mu}+A^{1-\mu} X B^{\mu}\right\|\right|+2\left|\left\|A^{\frac{1}{2}} X B^{\frac{1}{2}}\right\|\right|\right) \\
& \leq\left|\left\|A^{\mu} X B^{1-\mu}+A^{1-\mu} X B^{\mu}\right\|\right| .
\end{aligned}
$$

Since

$$
\begin{aligned}
& \lim _{\mu \rightarrow \frac{1}{2}} \frac{1}{|1-2 \mu|}\left|\int_{\mu}^{1-\mu}\right|\left\|A^{v} X B^{1-v}+A^{1-v} X B^{v}\right\||d v| \\
& =\lim _{\mu \rightarrow \frac{1}{2}} \frac{1}{2}\left(\left|\left\|A^{\mu} X B^{1-\mu}+A^{1-\mu} X B^{\mu}\right\|\right|+2\left|\left\|A^{\frac{1}{2}} X B^{\frac{1}{2}}\right\|\right|\right) \\
& =2\left|\left\|A^{\frac{1}{2}} X B^{\frac{1}{2}}\right\|\right|,
\end{aligned}
$$

the inequalities in (2.1) follow by combining (2.2) and (2.3).

Applying the previous lemma to the function $f(v)=\left\|\mid A^{v} X B^{1-v}+A^{1-v} X B^{v}\right\| \|$ on the interval $\left[\mu, \frac{1}{2}\right]$ when $0 \leq \mu \leq \frac{1}{2}$, and on the interval $\left[\frac{1}{2}, \mu\right]$ when $\frac{1}{2} \leq \mu \leq 1$, we obtain the following.

Theorem 2. Let $A, B, X$ be operators such that $A, B$ are positive. Then for $0 \leq \mu \leq 1$ and for every unitarily invariant norm, we have

$$
\begin{aligned}
& \left|\left\|A^{\frac{2 \mu+1}{4}} X B^{\frac{3-2 \mu}{4}}+A^{\frac{3-2 \mu}{4}} X B^{\frac{2 \mu+1}{4}}\right\|\right| \\
& \leq \frac{2}{|1-2 \mu|}\left|\int_{\mu}^{\frac{1}{2}}\right|\left\|A^{v} X B^{1-v}+A^{1-v} X B^{v}\right\||d v| \\
& \leq \frac{1}{4}\left(\left|\left\|A^{\mu} X B^{1-\mu}+A^{1-\mu} X B^{\mu}\right\|\right|+2\left|\left\|A^{\frac{2 \mu+1}{4}} X B^{\frac{3-2 \mu}{4}}+A^{\frac{3-2 \mu}{4}} X B^{\frac{2 \mu+1}{4}}\right\|\right|+\left|\left\|A^{\frac{1}{2}} X B^{\frac{1}{2}}\right\|\right|\right) \\
& \leq \frac{1}{2}\left(\left|\left\|A^{\mu} X B^{1-\mu}+A^{1-\mu} X B^{\mu}\right\|\right|+2\left|\left\|A^{\frac{1}{2}} X B^{\frac{1}{2}}\right\|\right|\right) .
\end{aligned}
$$


The inequality (2.4) and the first inequality in (1.1) yield the following refinement of the first inequality in (1.1).

Corollary 1. Let $A, B, X$ be operators such that $A, B$ are positive. Then for $0 \leq \mu \leq 1$ and for every unitarily invariant norm, we have

$$
\begin{aligned}
& 2\left|\left\|A^{\frac{1}{2}} X B^{\frac{1}{2}}\right\|\right| \\
& \leq||\left|A^{\frac{2 \mu+1}{4}} X B^{\frac{3-2 \mu}{4}}+A^{\frac{3-2 \mu}{4}} X B^{\frac{2 \mu+1}{4}} \|\right| \\
& \leq \frac{2}{|1-2 \mu|}\left|\int_{\mu}^{\frac{1}{2}}\right|\left\|A^{v} X B^{1-v}+A^{1-v} X B^{v}\right\||d v| \\
& \leq \frac{1}{4}\left(\left|\left\|A^{\mu} X B^{1-\mu}+A^{1-\mu} X B^{\mu}\right\|\right|+2\left|\left\|A^{\frac{2 \mu+1}{4}} X B^{\frac{3-2 \mu}{4}}+A^{\frac{3-2 \mu}{4}} X B^{\frac{2 \mu+1}{4}}\right\|\right|+\left|\left\|A^{\frac{1}{2}} X B^{\frac{1}{2}}\right\|\right|\right) \\
& \leq \frac{1}{2}\left(\left|\left\|A^{\mu} X B^{1-\mu}+A^{1-\mu} X B^{\mu}\right\|\right|+2\left|\left\|A^{\frac{1}{2}} X B^{\frac{1}{2}}\right\|\right|\right) . \\
& \leq\left|\left\|A^{\mu} X B^{1-\mu}+A^{1-\mu} X B^{\mu}\right\|\right| .
\end{aligned}
$$

Applying the previous lemma to the function $f(v)=\left\||| A^{v} X B^{1-v}+A^{1-v} X B^{v}\right\| \mid$ on the interval $[0, \mu]$ when $0 \leq \mu \leq \frac{1}{2}$, and on the interval $[\mu, 1]$ when $\frac{1}{2} \leq \mu \leq 1$, we obtain the following theorem.

Theorem 3. Let $A, B, X$ be operators such that $A, B$ are positive. Then

(1) for $0 \leq \mu \leq \frac{1}{2}$ and for every unitarily norm,

$$
\begin{aligned}
& \left|\left\|A^{\frac{\mu}{2}} X B^{1-\frac{\mu}{2}}+A^{1-\frac{\mu}{2}} X B^{\frac{\mu}{2}}\right\|\right| \\
& \leq \frac{1}{\mu} \int_{0}^{\mu}\left|\left\|A^{v} X B^{1-v}+A^{1-v} X B^{v}\right\|\right| d v \\
& \leq \frac{1}{4}\left(|\|A X+X B\||+2\left|\left\|A^{\frac{\mu}{2}} X B^{1-\frac{\mu}{2}}+A^{1-\frac{\mu}{2}} X B^{\frac{\mu}{2}}\right\|\right|+\left|\left\|A^{\mu}+X B^{1-\mu}+A^{1-\mu} X B^{\mu}\right\|\right|\right) \\
& \leq \frac{1}{2}\left(|\|A X+X B\||+\left|\left\|A^{\mu}+X B^{1-\mu}+A^{1-\mu} X B^{\mu}\right\|\right|\right) .
\end{aligned}
$$

(2) for $\frac{1}{2} \leq \mu \leq 1$ and for every unitarily norm,

$$
\begin{aligned}
& \left|\left\|A^{\frac{1+\mu}{2}} X B^{\frac{1-\mu}{2}}+A^{\frac{1-\mu}{2}} X B^{\frac{1+\mu}{2}}\right\|\right| \\
& \leq \frac{1}{1-\mu} \int_{\mu}^{1}\left|\left\|A^{v} X B^{1-v}+A^{1-v} X B^{v}\right\|\right| d v \\
& \leq \frac{1}{4}\left(|\|A X+X B\||+2\left|\left\|A^{\frac{1+\mu}{2}} X B^{\frac{1-\mu}{2}}+A^{\frac{1-\mu}{2}} X B^{\frac{1+\mu}{2}}\right\|\right|+\left|\left\|A^{\mu} X B^{1-\mu}+A^{1-\mu} X B^{\mu}\right\|\right|\right) \\
& \leq \frac{1}{2}\left(|\|A X+X B\||+\left|\left\|A^{\mu} X B^{1-\mu}+A^{1-\mu} X B^{\mu}\right\|\right|\right) .
\end{aligned}
$$

Since the function $f(v)=\left\||| A^{v} X B^{1-v}+A^{1-v} X B^{v} \mid\right\|$ is decreasing on the interval $\left[0, \frac{1}{2}\right]$ and increasing on the interval $\left[\frac{1}{2}, 1\right]$, and using the inequalities (2.6) and (2.7), we obtain the refinement of the second inequality in (1.1). 
Corollary 2. Let $A, B, X$ be operators such that $A, B$ are positive. Then for $0 \leq \mu \leq 1$ and for every unitarily invariant norm, we have

(1) for $0 \leq \mu \leq \frac{1}{2}$ and for every unitarily norm,

$$
\begin{aligned}
& \left|\left\|A^{\mu} X B^{1-\mu}+A^{1-\mu} X B^{\mu}\right\|\right| \\
& \leq\left|\left\|A^{\frac{\mu}{2}} X B^{1-\frac{\mu}{2}}+A^{1-\frac{\mu}{2}} X B^{\frac{\mu}{2}}\right\|\right| \\
& \leq \frac{1}{\mu} \int_{0}^{\mu}\left|\left\|A^{v} X B^{1-v}+A^{1-v} X B^{v}\right\|\right| d v \\
& \leq \frac{1}{4}\left(|\|A X+X B\||+2\left|\left\|A^{\frac{\mu}{2}} X B^{1-\frac{\mu}{2}}+A^{1-\frac{\mu}{2}} X B^{\frac{\mu}{2}}\right\|\right|+\left|\left\|A^{\mu} X B^{1-\mu}+A^{1-\mu} X B^{\mu}\right\|\right|\right) \\
& \leq \frac{1}{2}\left(|\|A X+X B\||+\left|\left\|A^{\mu} X B^{1-\mu}+A^{1-\mu} X B^{\mu}\right\|\right|\right) . \\
& \leq|\|A X+X B\|| .
\end{aligned}
$$

(2) for $\frac{1}{2} \leq \mu \leq 1$ and for every unitarily norm,

$$
\begin{aligned}
& \left|\left\|A^{\mu} X B^{1-\mu}+A^{1-\mu} X B^{\mu}\right\|\right| \\
& \leq\left|\left\|A^{\frac{1+\mu}{2}} X B^{\frac{1-\mu}{2}}+A^{\frac{1-\mu}{2}} X B^{\frac{1+\mu}{2}}\right\|\right| \\
& \leq \frac{1}{1-\mu} \int_{\mu}^{1}\left|\left\|A^{v} X B^{1-v}+A^{1-v} X B^{v}\right\|\right| d v \\
& \leq \frac{1}{4}\left(|\|A X+X B\||+2\left|\left\|A^{\frac{1+\mu}{2}} X B^{\frac{1-\mu}{2}}+A^{\frac{1-\mu}{2}} X B^{\frac{1+\mu}{2}}\right\|\right|+\left|\left\|A^{\mu} X B^{1-\mu}+A^{1-\mu} X B^{\mu}\right\|\right|\right) \\
& \leq \frac{1}{2}\left(|\|A X+X B\||+\left|\left\|A^{\mu} X B^{1-\mu}+A^{1-\mu} X B^{\mu}\right\|\right|\right) \\
& \leq|\|A X+X B\|| .
\end{aligned}
$$

It should be noticed that in the inequalities (2.6) to (2.9),

$$
\begin{aligned}
& \lim _{\mu \rightarrow 0} \frac{1}{\mu} \int_{0}^{\mu}\left|\left\|A^{v} X B^{1-v}+A^{1-v} X B^{v}\right\|\right| d v \\
& \lim _{\mu \rightarrow 1} \frac{1}{1-\mu} \int_{\mu}^{1}\left|\left\|A^{v} X B^{1-v}+A^{1-v} X B^{v}\right\|\right| d v \\
& =|\|A X+X B\|| .
\end{aligned}
$$

\section{Acknowledgements}

This article is prepared before the author's visit to Udine University, he wishes to express his gratitude to Prof. Corsini, Dr. Paronuzzi and Prof. Russo for their hospitality. Also, he wishes to thank Mr. Baojie Zhang, from Qujing Normal University, for the discussion. This research is financed by CMEC (KJ091104, KJ111107), CSTC, CTGU (10QN-27) and QNU (2008QN-034).

\section{Competing interests}

The authors declare that they have no competing interests.

\section{Received: 1 October 2011 Accepted: 27 January 2012 Published: 27 January 2012}

\section{References}

1. Bhatia, R, Davis, C: More matrix forms of the arithmeticgeometric mean inequality. SIAM J Matrix Anal Appl. 14, 132-136 (1993). doi:10.1137/0614012

2. Kittaneh, F: On the convexity of the Heinz means. Integ Equ Oper Theory. 68, 519-527 (2010). doi:10.1007/s00020-0101807-6 
3. Bullen, PS: A Dictionary of Inequalities. In Pitman Monographs and Surveys in Pure and Applied Mathematics, vol. 97, Addison Wesley Longman Ltd., U.K (1998)

doi:10.1186/1029-242X-2012-18

Cite this article as: Feng: Refinements of the Heinz inequalities. Journal of Inequalities and Applications 2012

2012:18.

Submit your manuscript to a SpringerOpen ${ }^{\circ}$ journal and benefit from:

- Convenient online submission

- Rigorous peer review

- Immediate publication on acceptance

- Open access: articles freely available online

- High visibility within the field

- Retaining the copyright to your article

Submit your next manuscript at $\boldsymbol{\nabla}$ springeropen.com 